\title{
Ex-Vivo Comparison of the Dentin Remov- al Ability of One Shape and F6 SkyTaper Rotary Files
}

\section{Z Khalilak ${ }^{1}$, I Sattarian², S Tour Savadkouhi ${ }^{2}$ *1}

1- Assistant Professor, Endodontics Dept, Member Ship of Dental Material Research Center, Faculty of Dentistry, Tehran Medical Sciences, Islamic Azad University, Tehran, Iran

2- Dentist, Tehran, Iran.

\begin{tabular}{l} 
ARTICLE INFO \\
Article History \\
Received: August 2019 \\
Accepted: Sep 2019 \\
ePublished: Nov 2019 \\
\\
\hline Corresponding author: \\
S Tour Savadkouhi,Assis- \\
tant Professor, End- \\
odontics Dept, Faculty \\
of Dentistry, Tehran \\
Medical Sciences, Is- \\
lamic Azad University, \\
Tehran, Iran \\
Email: s_savadkouhi@ \\
yahoo.com
\end{tabular}

\begin{abstract}
Background and Aim: One of the important characteristics of nickel-titanium (NiTi) rotary files is their dentin removal ability. The purpose of this study was to compare One Shape and F6 SkyTaper files in terms of dentin removal from the mesiobuccal canals of extracted first mandibular molars.

Materials and Methods:In this experimental study, 24 mesiobuccal canals of extracted first mandibular molars were selected. After preparing an access cavity, the samples were divided into two groups $(\mathrm{n}=12)$. The samples were air-dried and incubated for 8 hours at $82^{\circ} \mathrm{C}$ for moisture removal. The initial weight of the specimens was then measured. The samples in group A were prepared using One Shape (\#25/0.06) files while the samples in group B were prepared using F6 SkyTaper (\#25/0.06) files at a speed of 400 revolutions per minute (rpm) and torque of $2.2 \mathrm{~N} / \mathrm{cm}$ for 10 seconds. The samples were again rinsed, dried, and incubated to eliminate moisture. The weights of the teeth were measured after preparation to compare the dentin removal efficacy. The results were analyzed using paired t-test $(\mathrm{P}<0.05)$.

Results: The mean weight of the removed dentin was $0.053 \pm 0.019 \mathrm{~g}$ in the SkyTaper F6 group and $0.045 \pm 0.073 \mathrm{~g}$ in the One Shape group, which was $0.008 \mathrm{~g}$ or $15 \%$ less in the One Shape group, but the difference was not statistically significant $(\mathrm{P}=0.6)$. Conclusion: The amount of the dentin removed from the root canals by the SkyTaper F6 rotary system was greater compared to the One Shape rotary system although the difference was not statistically significant.

Keywords: Dentin, Endodontics, Equipment Design, Root Canal Preparations, Nickel, Titanium
\end{abstract}

J Res Dent maxillofac Sci 2019;4(4):6-10.

DOI: $10.29252 / j r d m s .4 .4 .6$

\section{Introduction:}

The most important step in a successful endodontic treatment involves a proper cleaning and shaping of root canals. The purpose of root canal preparation is to remove the entire infected necrotic or inflamed tissue remnants and to create a tapered canal to facilitate irrigation and obturation. ${ }^{(1)}$
Files are the most important instruments for cleaning and shaping the root canals. Today, nickel-titanium (NiTi) rotary files are popular due to their flexibility and resistance to fatigue. $^{(2-4)}$ These files can safely remove dentin in a very short time, even in curved root canals. ${ }^{(5,6)}$ 
The dentin removal ability is one of the most important features of a file, which depends on the number of flutes, the cross-section design, the geometric design, the tip design, dentin chip removal capability, alloy properties, and helical and rake angles. ${ }^{(7-14)}$

The F6 SkyTaper System (Komet Brasseler, Lemgo, Germany) is a rotary single file system. These files have a $6 \%$ constant taper in five sizes $(\# 20, \# 25, \# 30, \# 35$, and \#40) and are available in three lengths of 21,25 , and $31 \mathrm{~mm}$. These files have a semi-cutting tip and a double $\mathrm{S}$ cross-section. They should be used with a torque of 2.2 $\mathrm{N} / \mathrm{cm}$ and speeds of 300 to 500 revolutions per minute (rpm) for \#20, \#25, and \#30. ${ }^{(14)}$

The One Shape Rotary File System (Micro Mega, Besancon, France) is a fifth-generation single rotary file system. These files have a size of 25 , a taper of $6 \%$, and a length of $25 \mathrm{~mm}$. According to the manufacturer's instructions, rotary machines at speeds of 350-450 rpm and a maximum torque of $2.5 \mathrm{~N} / \mathrm{cm}$ are proposed for these files. One Shape files have a non-cutting safety tip, which helps its easy guide to the apex of the root canals. The file has three different asymmetric cross-sections. There are three cutting edges in the first region, gradually changing from 3 to 2 cutting edges in the second region, and 2 cutting edges in the third area (the coronal part of the file). The asymmetric design makes the file more flexible. ${ }^{(14-21)}$

The dentin removal ability is measured based on measuring the weight of dental specimens or resin blocks before and after applying files for a specific period. ${ }^{(7-9,10)}$

Since only one study on the comparison of One Shape and F6 SkyTaper rotary files in terms of their dentin removal ability using AutoCAD was found, this study aimed to compare the One Shape and F6 SkyTaper files in terms of dentin removal from the mesiobuccal canals of extracted first mandibular molars.

\section{Materials and Methods}

In this ex-vivo experimental study, 24 extracted first mandibular molars were collected and disinfected in $5.25 \%$ sodium hypochlorite $(\mathrm{NaO}-$
$\mathrm{Cl})$ for 24 hours. Then, they were well rinsed and placed in normal saline until further interventions.

At first, radiographs were taken, and teeth with internal or external resorption in the mesial root were excluded from the study, whereas teeth with mature apices and no cracks, fractures, or decay on the root surface were included in the study.

After preparing an access cavity, the mesial canals were negotiated using two \#10 K-files (Dentsply, Maillefer, Switzerland) that were placed simultaneously in the canals to the tip of the apex. A separate apical foramen for each canal was confirmed, and teeth that did not meet this condition were excluded from the study.

To determine the curvature of the canal, radiographs were taken using the parallel technique directly from the buccolingual direction while the \#10 K-files were in the mesiobuccal root canals. The curvature of the mesiobuccal canal was measured according to the Schneider's technique, and the teeth with root curvatures ranging from 20 to 35 degrees and working lengths of about 20 to 22 $\mathrm{mm}$ were included in the study. ${ }^{(17,22)}$

The teeth were then washed with tap water, dried using compressed airflow, and incubated (Zenith Lab., DZF 6020, China) at $82^{\circ} \mathrm{C}$ for 6 hours to eliminate moisture. ${ }^{(15)}$ Then, the samples were divided into two groups (A and B) of 12 each, and the net weight of each tooth was measured using a digital scale (A\&D, GF 300, Germany) with the accuracy of 10-3. The numbers were recorded in datasheets. The samples were then kept in normal saline until the next intervention. ${ }^{(15)}$

In the next step, to prevent tooth movement during the preparation process, we placed each tooth inside gypsum molds up to the cementoenamel junction (CEJ).

The teeth in group A were prepared using \#25/6\% taper One Shape (Micro Mega, Besancon, France) files, whereas teeth in group B were prepared using \#25/6\% taper F6 SkyTaper (Komet Brasseler, Lemgo, Germany) files. 
Each root canal was irrigated with $3 \mathrm{ml}$ of normal saline using a 25-gauge needle tip (Supa Co., Tehran, Iran), which was passively $2 \mathrm{~mm}$ shorter than the working length. Files of both groups were used according to the manufacturer's instructions with a torque of $2.2 \mathrm{~N} / \mathrm{cm}$ and a speed of $400 \mathrm{rpm}$ for 10 seconds. Each file was used only to prepare one canal. The files were used with the DentaPort ZX rotary motor (J. Morita Corp., Kyoto, Japan). ${ }^{(19)}$

The teeth were then removed from the gypsum molds and washed under running water. Each dental canal was washed with $10 \mathrm{ml}$ of distilled water using a syringe with a 25 -gauge needle tip, then dried with compressed air, and incubated at $82^{\circ} \mathrm{C}$ for 6 hours to eliminate moisture.

Again, the weight of the teeth in each group was measured after the preparations by the files to measure the dentin removal rate from the canals within 10 seconds using a digital scale. ${ }^{(15)}$

Paired t-test was used for statistical comparison between the two groups $(\mathrm{P}<0.05)$.

\section{Result:}

This study was performed on the mesiobuccal canals of 24 teeth in two groups of One Shape and F6 SkyTaper.

The initial weight of the first mandibular molars, the weight after the preparation by the files, and the amount of the removed dentin are presented in Table 1.
The primary weights of the teeth were similar in the two groups, and their difference was not statistically significant $(\mathrm{P}=0.9)$.

The dentin removal rate was $0.053 \pm 0.019 \mathrm{~g}$ in the F6 SkyTaper group and $0.045 \pm 0.073 \mathrm{~g}$ in the One Shape group; the difference was statistically insignificant $(\mathrm{P}=0.6)$.

\section{Discussion:}

The purpose of this study was to compare two rotary file systems, namely One Shape and F6 SkyTaper, regarding their dentin removal ability from root canals.

Different methods are available to examine the ability of files to remove dentin, including the comparison of changes in the root canal volume and residual dentin thickness, ${ }^{(16,20)}$ the comparison of the weight loss of samples before and after filing, ${ }^{(15,19)}$ as well as the comparison of the penetration depth of the files into the samples. ${ }^{(17,19)}$

In this study, the dentin removal rate was measured by the weight loss method because weight measurement is one of the easiest and most accurate methods.

The files examined in this study were both from the fifth-generation single-file systems made from the Austenite 55-NiTi alloy with a size of 25 and a $6 \%$ taper with full rotational motions. The benefits of these systems make them easy to work with, save time, reduce the chance of file fracture, and prevent the spread of infection.

Table 1: Mean and standard deviation (SD) of the removed dentin in the experimental groups

\begin{tabular}{cccc}
\hline $\begin{array}{c}\text { Experimental } \\
\text { groups }\end{array}$ & Initial weight (g) & $\begin{array}{c}\text { Post-preparation } \\
\text { weight }(\mathrm{g})\end{array}$ & $\begin{array}{c}\text { Weight of the } \\
\text { removed dentin }(\mathrm{g})\end{array}$ \\
\hline F6 SkyTaper $(\mathrm{n}=12)$ & $1.68 \pm 0.26$ & $1.62 \pm 0.25$ & $0.053 \pm 0.019$ \\
\hline One Shape $(\mathrm{n}=12)$ & $1.72 \pm 0.31$ & $1.67 \pm 0.32$ & $0.045 \pm 0.073$ \\
\hline P-value & $\mathrm{P}=0.9$ & $\mathrm{P}=0.8$ & $\mathrm{P}=0.6$ \\
\hline
\end{tabular}


In the present study, the dentin removal rate was calculated based on the comparison of the weight difference between the initial and secondary weights of the samples after preparation by files for 10 seconds. The F6 SkyTaper files removed $0.008 \mathrm{~g}$ or $15 \%$ more dentin compared to the One Shape system although this difference was not statistically significant $(\mathrm{P}=0.6)$.

Although the files studied in this research have been investigated in various studies using various methods, the results of most of them are similar to the results of our study.

Rubio et al performed a study to determine the rate of root canal preparations of 10 types of rotary files. ${ }^{(23)}$ They compared the images before and after the preparation of the samples using the AutoCAD software. The results showed that F6 SkyTaper files were capable of removing $694.0 \pm 0.02 \mathrm{~mm} 2$ of dentin, but the dentin removal rate was $0.604 \pm 0.66 \mathrm{~mm} 2$ for One Shape files. ${ }^{(23)}$ Based on the results of this research, the dentin removal rate was higher for F6 SkyTaper files although there were no statistically significant differences $(\mathrm{P}<0.05)$. The results of the mentioned study were similar to the results of the present study.

In another study by Saleh and Rashid, comparisons were made among four single file systems, namely Wave One, One Shape, RECIPROC, and F360 files, using 64 resin blocks. ${ }^{(24)}$ According to the results of resin removal from the samples, the Wave One and RECIPROC were more effective than the One Shape and F360 files due to the reciprocal movements of the Wave One and RECIPROC files. ${ }^{(24)}$ The F360 system has the lowest cutting rate among other groups, which is due to the $4 \%$ taper. Of course, given that their research involved resin blocks, the results cannot be generalized to natural teeth.

Dagna et al studied F6 SkyTaper and F360 files to determine the amount of debris removal from 20 teeth that were divided into two groups of 12 samples each. ${ }^{(25)}$ The results showed that in the apical and coronal areas, both groups were the same, but in the middle zone of the canals, the F6 SkyTaper group showed a higher dentine removal rate. ${ }^{(25)}$ According to the results of the cited study, although both systems had the same characteristics in terms of alloy and design, the 6\% taper of the F6 SkyTaper files causes a higher rate of dental tissue removal compared to the $4 \%$ taper of the F360 files. ${ }^{(25)}$

According to the results of this study and similar research, there were no significant differences in dentin removal rates between the two experimental groups, but the dentin removal rate in the F6 SkyTaper group was about 15\% greater than that of the One Shape group. The reasons for the superiority of F6 SkyTaper files in dentin removal rates compared to One Shape files can be their different designs while the size and taper (\#25 and 6\%) are the same in both files. Among the differences in the design, the presence of a semi-cutting tip in F6 SkyTaper files, the thinnest design of the central core, and cutting blades with greater prominence and further intervals allow more dentin chip removal.

In the One Shape files, the non-cutting tip, thicker central core, and less prominent cutting blades make the file less cutting.

This study was done on human teeth to simulate clinical conditions. Although in some studies, resin blocks have been used, this method has some disadvantages, such as the difference in the hardness of resin blocks and dentin, as well as the dimensional changes in resin blocks due to the heat generated secondary to the rotation of the files.

None of the files were broken in the present study due to the use of a new file for each root canal.

\section{Conclusion:}

Based on the results of this study, $15 \%$ more dentin was removed from dental root canals with F6 SkyTaper rotary files compared to the One Shape rotary group although the difference was not statistically significant.

\section{References:}

1. Tomson PL, Simon SR. Contemporary cleaning and shaping of the root canal system. Prim Dent J. 2016 May 1;5(2):46-53.

2. Peters OA, Paque F. Current developments in rotary root canal instrument technology and clinical use: a review. Quintessence Int. 2010 Jun;41(6):479-88.

3. Peters OA, Schonenberger K, Laib A. Effects of four Ni-Ti preparation techniques on root canal geometry assessed by micro computed to- 
mography. Int Endod J. 2001 Apr;34(3):221-30. 4.Pettiette MT, Delano EO, Trope M. Evaluation of success rate of endodontic treatment performed by students with stainless-steel K-files and nickel-titanium hand files. J Endod. 2001 Feb;27(2):124-7.

5.Bergmans L, Van Cleynenbreugel J, Wevers M, Lambrechts P. Mechanical root canal preparation with NiTi rotary instruments: rationale, performance and safety. Status report for the American Journal of Dentistry. Am J Dent. 2001 Oct;14(5):324-33.

6.Peters OA. Current challenges and concepts in the preparation of root canal systems: a review. J Endod. 2004 Aug;30(8):559-67.

7.Vinothkumar TS, Miglani R, Lakshminarayananan L. Influence of deep dry cryogenic treatment on cutting efficiency and wear resistance of nickel-titanium rotary endodontic instruments. J Endod. 2007 Nov;33(11):1355-8.

8.Rapisarda E, Bonaccorso A, Tripi TR, Fragalk I, Condorelli GG. The effect of surface treatments of nickel-titanium files on wear and cutting efficiency. Oral Surg Oral Med Oral Pathol Oral Radiol Endod. 2000 Mar;89(3):363-8.

9.Schäfer E, Erler M, Dammaschke T. Comparative study on the shaping ability and cleaning efficiency of rotary Mtwo instruments. Part 1. Shaping ability in simulated curved canals. Int Endod J. 2006 Mar;39(3):196-202.

10.Bürklein S, Benten S, Schäfer E. Shaping ability of different single-file systems in severely curved root canals of extracted teeth. Int Endod J. 2013 Jun;46(6):590-7.

11.Berman MH. Cutting efficiency in complete coverage preparation. J Am Dent Assoc. 1969 Nov;79(5):1160-7.

12.Schäfer E, Oitzinger M. Cutting efficiency of five different types of rotary nickel-titanium instruments. J Endod. 2008 Feb;34(2):198-200.

13.Rzhanov EA, Belyaeva TS. Design features of rotary root canal instruments. ENDO-Endodontic Practice Today. 2012 Spring;6(1):29-39.

14.Agarwal S, Nagpal R, Singh UP. NiTi Endodontics: Contemporary Views Reviewed. Austin J Dent. 2018;5(4):1112.

15.Kataia MM, Kataia EM. Cutting efficiency of different cross sectional design protaperrotary instruments - in-vitro study. JIPBS. 2016;3(1):116122.
16. Alattar S, Nehme W, Diemer F, Naaman A. The influence of brushing motion on the cutting behavior of 3 reciprocating files in oval-shaped canals. J Endod. 2015 May;41(5):703-9.

17. Plotino G, Giansiracusa Rubini A, Grande NM, Testarelli L, Gambarini G. Cutting efficiency of Reciproc and waveOne reciprocating instruments. J Endod. 2014 Aug;40(8):1228-30.

18. Giansiracusa Rubini A, Plotino G, Al-Sudani D, Grande NM, Sonnino G, Putorti E, et al. A new device to test cutting efficiency of mechanical endodontic instruments. Med Sci Monit. 2014 Mar 6;20:374-8.

19. Moraes SH, Hancalves M, Filho MT, Filho IB. Cutting ability of nickel-titanium rotary systems ProTaper, Mtwo and K3. RSBO. 2012 AprJun;9(2):177-82.

20. Fayyad DM, Elhakim Elgendy AA. Cutting efficiency of twisted versus machined nickel-titanium endodontic files. J Endod. 2011 Aug;37(8):1143-6.

21. Cecchin D, de Sousa-Neto MD, Pecora JD, Gariba-Silva R. Cutting efficiency of four different rotary nickel: Titanium instruments. J Conserv Dent. 2011 Apr;14(2):117-9.

22. Schneider SW. A comparison of canal preparations in straight and curved root canals. Oral Surg Oral Med Oral Pathol. 1971 Aug;32(2):2715.

23. Rubio J, Zarzosa JI, Pallarés A. Comparison of Shaping Ability of 10 Rotary and Reciprocating Systems: an In Vitro Study with AutoCad. Acta Stomatol Croat. 2017 Sep;51(3):207-16.

24. Saleh ARM, Rashid AA. Canal Central Ability of Four Different Endodontic Single-File Systems in Simulated L-Shaped Resin Canals. Int J Dent Oral Health. 2016 Jul;2(7):doi http://dx.doi. org/10.16966/2378-7090.213.

25. Dagna A, Gastaldo G, Beltrami R, Chiesa M, Poggio C. F360 and F6 Skytaper: SEM evaluation of cleaning efficiency. Ann Stomatol (Roma). 2016 Feb 12;6(3-4):69-74.

Please cite this paper as:

Khalilak Z, Sattarian I, Tour Savadkouhi S. ExVivo Comparison of the Dentin Removal Ability of One Shape and F6 SkyTaper Rotary Files. J Res Dentomaxillofac Sci. 2019; 4 (4) :6-10 